\title{
Duplex ultrasound evaluation for dialysis access selection and maintenance: a practical guide
}

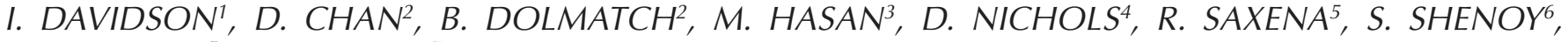 \\ M. VAZQUEZ ${ }^{5}$, M. GALLIENI
}

'Division of Transplant, Department of Surgery, Parkland Memorial Hospital University of Texas Southwestern Medical Center, Dallas - USA

${ }^{2}$ Division of Interventional Radiology, University of Texas Southwestern Medical Center, Dallas - USA

${ }^{3}$ Baptist Cardiac \& Vascular Institute, Miami, Florida - USA

${ }^{4}$ Vascular Center, Medical City Hospital Dallas - USA

${ }^{5}$ Division of Nephrology, Department of internal medicine, University of Texas Southwestern Medical Center, Dallas - USA

${ }^{6}$ Section of Transplantation, Department of Surgery, Washington University School of Medicine, St Louis - USA

${ }^{7}$ Renal Unit San, Paolo Hospital, University of Milano, Milano - Italy

\begin{abstract}
Detailed case directed history and examination is the mainstay of dialysis access modality selection, ie site and type of access, as well as for maintenance of dialysis access for longevity. As a logical step following history and physical examination, duplex ultrasound evaluation (DUE) is the most cost effective and non-invasive screening tool for evaluation for access placement and for assessment of an established access. Pre-operative vascular mapping allows selection of the optimal dialysis access modality and site. In established accesses, duplex ultrasound testing will diagnose the majority of vascular access complications and direct proper surgical or interventional radiology management. This review outlines a practical decision-making algorithm using DUE for choosing and managing the dialysis access. (J Vasc Access 2008; 9: 1-9)
\end{abstract}

Key words: Duplex ultrasound, Hemodialysis access, Algorithm, Vascular mapping, Hand ischemia

\section{INTRODUCTION}

A general review describing triage of end-stage renal disease (ESRD) patients for renal replacement therapy (RRT) was recently published (1). It emphasized the importance of long-term planning in choosing the best RRT option sequentially over a patient's lifetime. The use of peritoneal dialysis (PD) as the first treatment option, whenever feasible, was emphasized. However, most patients are treated by hemodialysis (HD), and vascular access planning is a crucial step in their path to dialysis treatment. This review outlines the practical use and limitations of DUE in access triage, when HD is the chosen mode of RRT.

\section{EVALUATION STEPS PRIOR TO ACCESS PLACEMENT}

Pertinent patient history and physical examination (H\&P) are by far the most important first steps in assessing the course of action, both prior to access placement and when evaluating an established access with problems. These basic skills serve as a common sense stepwise triage to more expensive and often invasive testing. A carefully performed $\mathrm{H} \& \mathrm{P}$ will yield proper patient selection for the most optimal dialysis modality and site of access placement. When the patient and family members are active participants in the decision-making process, adherence greatly improves $(2,3)$. The following paragraphs highlight the basic steps in obtaining a complete H\&P.

First, determine the most optimal type of modality of dialysis access, ie peritoneal dialysis (PD) vs hemodialysis (HD) (1). Often patients have little or no prior knowledge or understanding of treatment options, which complicates the initial (surgical) evaluation. We believe that nephrologists should spend more time with each patient explaining the importance of an early choice of dialysis modality, and consequently early dialysis access preparation (1). A pertinent history includes type and nature of past access procedures (especially central venous 
catheters (CVC), peripherally inserted central catheters (PICC) lines) and pacemakers, breast and axillary dissection surgery, chest radiation and emergency vascular cut-downs.

Physical examination includes a detailed search for veins in both upper extremities starting with the forearm cephalic and basilic veins. An upper arm tourniquet is placed to augment the veins and the patient is asked to make fists to dilate the veins.

The mainstay of surveillance and monitoring of access is physical examination. The presence of a palpable thrill, pulse quality, and appearance of the overlying skin (ie redness, edema, skin quality) are essential components of the examination. The H\&P determine if any further testing is needed.

Vascular examination must assess both the arterial (pulse quality, segmental blood pressure (BP), and the Allen test) (4) as well as the venous system, through inspection and palpation $(2,3)$. Visible veins are marked with an indelible pen to guide and help the DUE technologist and the surgeon during these examinations. After a thorough H\&P examination the surgeon will in most instances have a strong indication of site and type of access. Therefore, DUE is often used to confirm or correct this initial impression and to define surgical and interventional anatomy.

The physical examination must include and take into consideration the significance of scars, current and previous CVCs, past chest surgery (ie pacemakers), presence of edema and chest wall collateral vein formation suggesting central vein pathology.

A word of caution is warranted. Both physical and DUE examination may underestimate the presence and size of veins and arteries in an individual in a state of vasoconstriction, often seen in an anxious patient, in a cold examining room and when using cold ultrasound conductive gel. If this situation is suspected it is wise to reassess the patient before surgery in the pre-operative area where the patient is warm and relaxed.

\section{Duplex ultrasound evaluation}

DUE uses conventional B-Mode (black and white brightness) imaging with Doppler imaging (color and spectral analysis). Color Doppler imaging is displayed simultaneously on top of the B-Mode images using a computer process called auto-correlation; however, Doppler spectral analysis is displayed simultaneously as a separate image using a computer processor called Fast Fourier Transformer (FFT). Doppler imaging is generated only where Doppler energy (shift) is obtained while scanning a moving structure (in this case blood flowing in veins, arteries and in the dialysis access).
DUE is the most cost-effective non-invasive test for dialysis access planning. Nearly all patients requiring dialysis access should undergo DUE examination The DUE testing should be based upon prior H\&P. The quality of the DUE examination is operator dependent. Ideally, the surgeon should be present to direct the sequence of examination steps and mark the skin, documenting vessel size, intended surgery sites, and anatomical variations $(2,3)$. Table I lists the specific features assessed during DUE.

Surgery using a (radial) artery diameter of $\leq 2.5 \mathrm{~mm}$ will likely fail from inadequate fistula inflow $(<500$ $\mathrm{ml} / \mathrm{min}$ ), ie lack of maturation. An arteriovenous $(\mathrm{AV})$ anastomosis diameter of $\leq 2.5 \mathrm{~mm}$ is also likely to yield inadequate flow rate $(4,5)$ (Tab. II). DUE can also be used to assess blood flow problems asso-

TABLE I - MAIN FEATURES ADDRESSED BY DUPLEX DOPPLER VASCULAR EXAMINATION

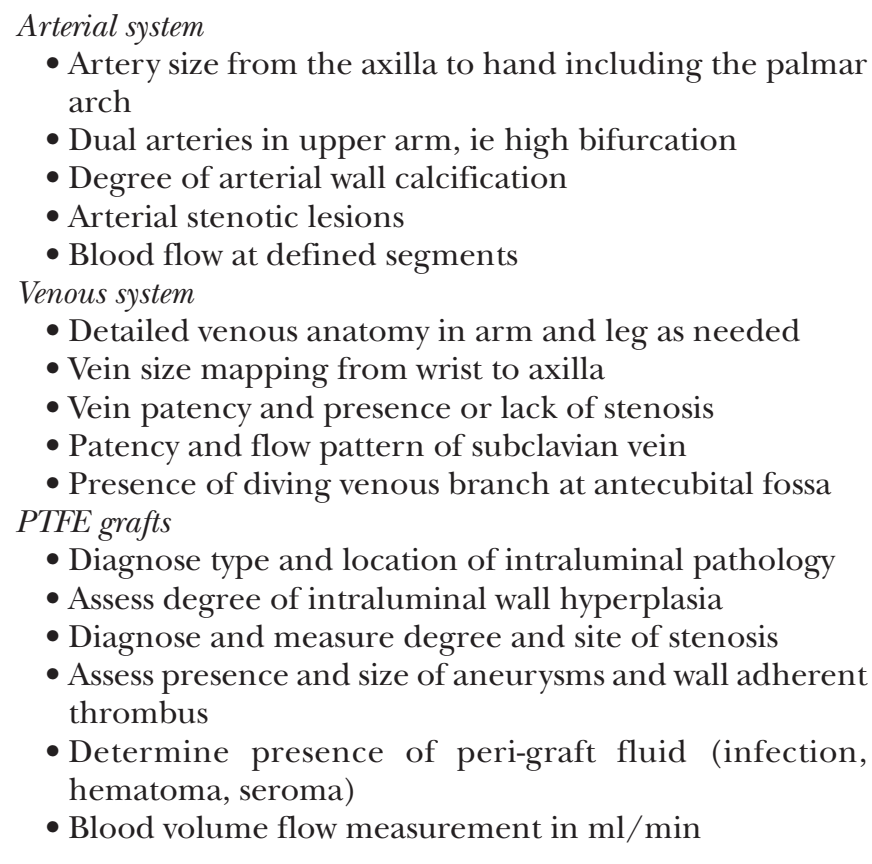

TABLE II - MINIMAL VASCULAR REQUIREMENTS FOR SUCCESSFUL AVF

1. Anastomosis luminal diameter of at least $2.5 \mathrm{~mm}$

2. Lack of segmental stenosis of artery or vein

3. Straight vein cannulation segment of at least $20 \mathrm{~cm}$

4. Vein cannulation segment less than $5 \mathrm{~mm}$ below skin surface

5. Matured vein (or PTFE graft) diameter of at least $6 \mathrm{~mm}$

6. Flow Rate of 500-600 mL/min or more

7. Absence of central vein obstruction

8. Segmental blood pressure differential of less than 20 $\mathrm{mm} \mathrm{Hg}$ 
ciated with established HD access. It will confirm the diagnosis and direct the treatment in the majority of cases. In a more complex access situation invasive imaging technology such as a fistulogram is warranted $(6,7)$. DUE and IV contrast fistulogram are not competitive but complementary. In most instances, when DUE examination suggests pathology, a fistulogram with simultaneous interventional treatment is indicated. The selection and order of tests may be dictated by local availability of equipment and personnel training and skills.

DUE examination should represent true teamwork between the ultrasound technologist and the surgeon. In the majority of cases this is the anatomic diagnostic examination dictating the type and site of the surgical access procedure.

\section{DUE examination for planning of a new dialysis access}

Table III summarizes the indications for pre-operative DUE are summarized in (8). Prior to DUE the surgeon will have seen the patient in the examining room. Based on history and physical examination, the surgeon will have a strong indication of the likely access site and type. Ideally the extremities have been marked for palpable veins and arterial pulses directing the ultrasound vascular technologist.

An established dialysis access may have multiple anatomical defects in up to one-third of cases. Therefore, the dialysis access evaluated for a specific symptom should also undergo complete assessment of the inflow artery, the access itself and the outflow vein including the subclavian vein patency and flow pattern.

\section{STEPWISE STRATEGY IN THE DUPLEX ULTRASOUND EVALUATION}

\section{Venous system preoperative evaluation}

Antecubital superficial veins. Both arms are examined starting with the non-dominant side. Place an upper arm tourniquet with the arm hanging down with the elbow slightly bent. Making hand fists over a soft ball helps dilate veins. Apply pre-warmed body temperature conducting gel. Avoid cold temperature in the examination room.

First, visualize and define the antecubital (AC) superficial vein anatomy (median cubital vein (MCV), cephalic vein $(\mathrm{CV})$, basilic vein $(\mathrm{BV})$ ) and their continuation proximally up in the arm and distally down the forearm. Although much variation exists, there is a typical venous anatomy pattern where the $\mathrm{CV}$ and the BV are connected by the MCV, depicted in Figure 1. Uniformly, a diving vein (vena communi-

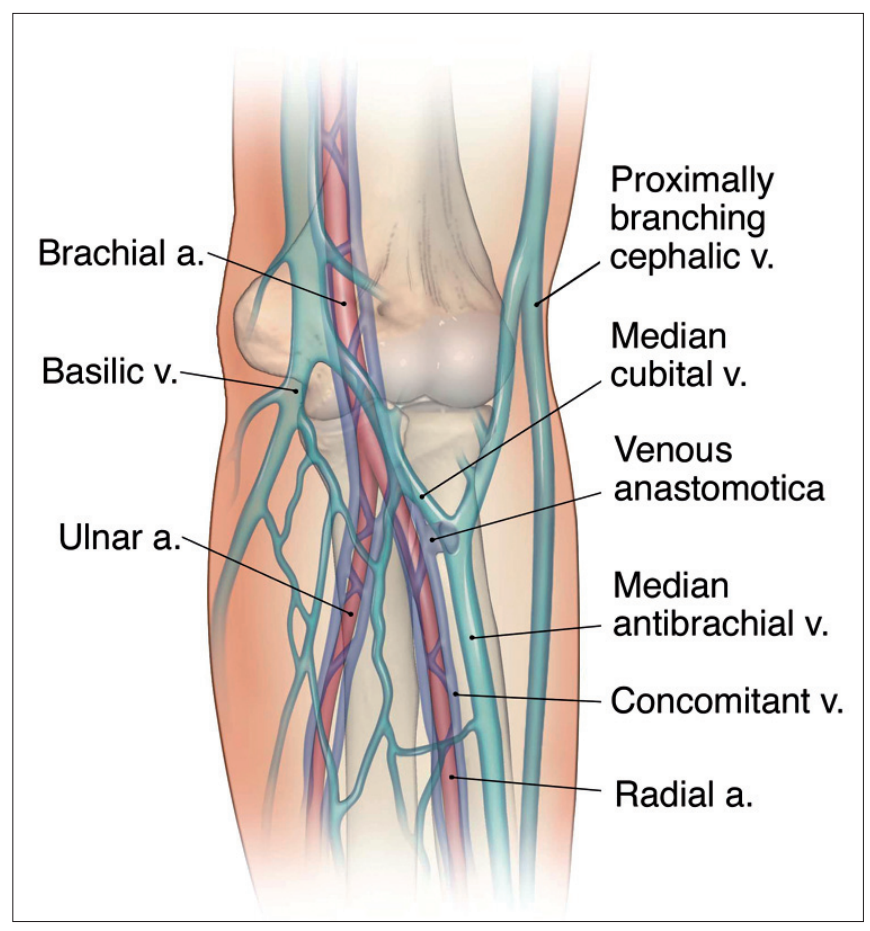

Fig. 1 - View of the antecubital fossa (AC) vascular anatomy, which may vary considerably. This image encompasses all common variations into one general overview. Anatomy atlases may also have different nomenclatures. Typically the forearm cephalic vein $(C V)$ bridges over to the basilic vein (CV) via the median antecubital vein. Sometimes this communicating vein is absent. The CV continues up on the upper arm and uniformly joins with the (true) cephalic vein at the lateral aspect $4-5 \mathrm{~cm}$ above the $A C$. The upper arm $\mathrm{CV}$ is unpredictable and may be too small or located too deep for successful cannulation AVF. The vena anastomotica (VA) connects the superficial $A C$ veins with one the deep concomitant brachial veins. The origin of the VA varies considerably. The level of brachial artery (BA) bifurcation is unpredictable. In 15-20\% there are duplicate arteries at the AClevel, with high bifurcation at any point in the upper arm or even at or above the axilla. The two arteries represent and will in the forearm become the more superficial radial artery $(R A)$ and the larger and deeper ulnar artery (UA). DUE will delineate and define the vascular anatomy for the best hemodialysis access site and type.

TABLE III - ABSOLUTE INDICATIONS FOR DULPEX DOPPLER EVALUATION OF DIALYSIS ACCESS PATIENTS

1. Extremity edema/swelling

2. Prominent/collateral veins on extremity

3. Size differential between extremities

4. History of central venous catheters

5. Previous access surgeries at, below or above the planned site

6. Previous surgeries on arm, neck or chest

cantes) at the AC level connects the superficial system to the deep brachial (concomitant) veins. In cases of inadequate forearm anatomy for a wrist ar- 
teriovenous fistula (AVF), this is the ideal site for the vein anastomosis forearm loop with a polytetrafluoroethylene (PTFE) graft, providing three or more outflow veins. Although usually present, the diving vein is variable and may connect to any of the superficial veins at the AC. Typically the CV and MCV communicate at the origin of the diving branch. DUE will define the antecubital fossa vascular anatomy and help plan the surgical site. In the absence of forearm vascular anatomy for an AVF at the wrist and with a small or absent upper arm CV, a forearm PTFE loop graft may be the preferred access choice. This will allow upper arm veins to grow and dilate making future upper arm access (ie basilic vein transposition, brachial-cephalic AVF and upper arm PTFE graft) successful. Imaging must document full compressibility and patency of all veins examined with absence of any luminal defects and/or thrombosis.

The forearm cephalic vein is followed from the antecubital fossa to the wrist. Several diameter measurements of the venous size at different levels are obtained and venous compression is performed throughout the examination to confirm patency. At the wrist a vein diameter of a least $2.5-3.0 \mathrm{~mm}$ is desirable. Note the presence of the cephalic vein dorsal branch. This bifurcation (if distal enough, or within 5-6 cm from the joint) is optimal for an end "patch" CV to side radial artery anastomosis (3). Sometimes there are duplicate CVs in close proximity or other anatomical findings (ie multiple branching) that would make future successful dialysis needle cannulation less likely. Common sense and judgment must prevail in the decision-making.

Next follow the upper arm cephalic vein toward the shoulder. There is often a lateral branch from the forearm (branch of the CV) joining the vein about 4-6 $\mathrm{cm}$ above the AC. In case of an upper arm brachial artery to CV AVF, this lateral branch may have to be divided or could be used depending on size, location and distance to the brachial artery. Sometimes a brachio-cephalic AVF at the elbow site may be created using the bifurcation of the diving vena communicantes as a vein "patch" anastomoisis to the side brachial artery. To avoid excess flow or "steal" syndrome the anastomosis is accomplished with a running suture limiting the diameter of the anastomosis to $4-5 \mathrm{~mm}$. An AVF at the AC site is the authors' second choice when suitable vascular anatomy at the wrist is lacking.

The basilic vein is identified either at the AC by following the MCV into the BV or by finding the BV more proximally closer to the axilla. Because of its deep location the BV ("the hidden vein") is usually untouched by veno-punctures (except for PICC lines). The authors are selective when choosing the upper arm BV transposition (BVT) procedure, since a PTFE graft may produce equal or better outcome than a borderline BV (5). It is optimal to have the entire upper arm BV length from AC to the axilla for a BVT procedure. The BV usually bifurcates $4-5 \mathrm{~cm}$ above the AC and sometimes into 3-4 branches, one of which becomes the forearm main BV. Sometimes the BV is missing or joins the brachial veins at the mid upper arm level. Although transposing a brachial vein, as a continuation in such a case is feasible, the authors generally advise against this. Also, a more distal PTFE (ie a fore arm loop) graft will make upper arm veins enlarge for future use.

A consistent and large $(3-5 \mathrm{~mm})$ forearm basilic vein is also suitable for transposition. Although an AVF can be made between the distal BV and the ulnar artery at the wrist, the awkward position makes long-term dialysis needle punctures practically difficult. When feasible the forearm BV is transposed in a straight or loop configuration to the distal radial artery at the wrist or to the brachial or radial artery at the AC fossa, respectively. The loop configuration is chosen when the wrist arteries are deemed inadequate.

The remainder of the upper extremity venous system (brachial, axillary, subclavian and brachiocephalic veins) is examined for patency and Doppler flow characteristics. Special consideration is given to both the subclavian and brachiocephalic veins as flow is often phasic with respiration. Comparison between the left and right sides is very useful to detect any unnoticed obstruction where Doppler flow characteristics may be abnormal.

\section{Arterial system preoperative evaluation}

Upper extremity arteries are evaluated by duplex for patency and Doppler flow wave form characteristics. Examination includes the Allen test and in certain cases digital pressures.

In the upper arm the subclavian, axillary, brachial, radial and ulnar arteries are assessed for patency and the presence of calcifications or stenosis. Doppler characteristic of upper extremity arterial flow prior to the creation of a HD access is that of high resistivity which will change to hyper-dynamic low resistive characteristics after creating the access. An AVF created with a severely calcified radial artery or a radial artery $<2.0-2.5 \mathrm{~mm}$ has a high failure rate because of insufficient inflow (non-maturing AVF). In our past experience about one-third of AVFs fail to mature requiring revisions or a new access. In a recent multicenter (877 patients) double-blind randomized study of upper extremity AVFs the matura- 


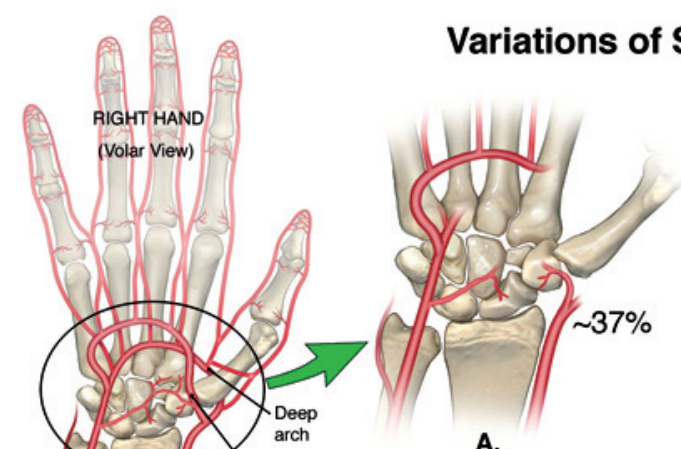

A.

Variations of Deep Palmar Arch

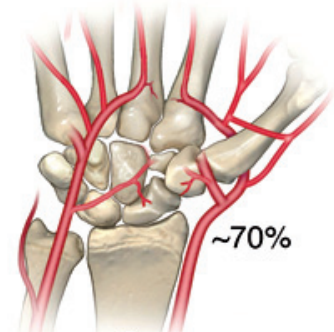

D.

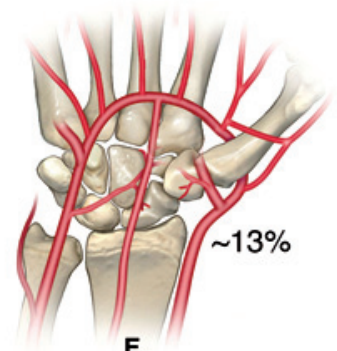

E.

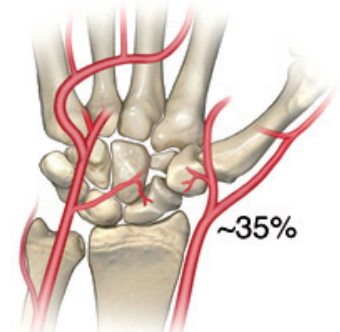

B.

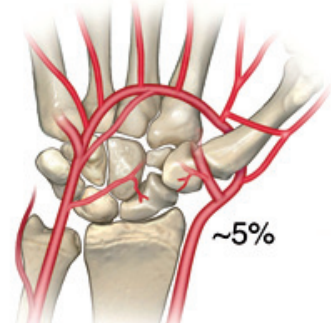

F.

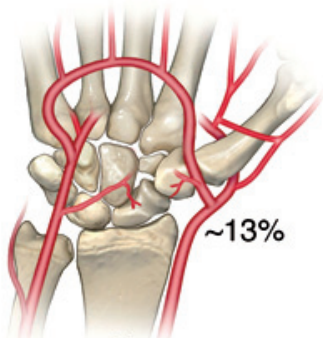

c.

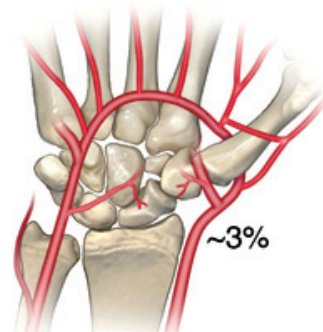

G.

Fig. 2 - The two palmar arterial arches are the likely major culprit for developing hand ischemia or steal syndrome after dialysis access placement. Depicted in this image are the two palmar arterial arches (deep and superficial) connecting the radial and ulnar arteries' flow into the hand. This ideal scenario is far from reality. The anatomical connections for both arches is quite variable and unpredictable as outlined in the inserted diagrams in the right and lower aspect of the image with approximate frequencies shown for each anatomical scenario. With only estimated 21 and $13 \%$ of the deep and superficial arches being completely connected, between the radial and ulnar arteries, respectively, it is not surprising that hand ischemia is a common outcome after dialysis access creation. In addition to the palmar arches anatomical prediction of hand ischemia, more proximal arterial patho-physiology, ie arterial stenosis or lack of generalized arterial blood flow adaptation (atherosclerosis) may be contributory factors in the development of symptomatic hand ischemia. DUE with and digital pressure measurement combined are the most helpful screening tests to evaluate, predict and prevent hand ischemic events after hemodialysis access placement.

tion failure was $59-62 \%(9)$.

Determining the ulnar artery size is an important part of the palmar arch assessment. A small or absent ulnar artery may increase the risk of steal syndrome. High bifurcation of the brachial artery, sometimes at or above the axilla, is seen in $15-20 \%$ of patients. This results in the (deeper and uniformly larger) ulnar and the (smaller and more superficial) radial artery into the forearm. If the AC level is the site for a PTFE graft or an artery-cephalic vein AVF, the larger and deeper ulnar artery is recommended for the anastomosis. In this dual artery situation the smaller radial artery serves as collateral circulation representing a "partial" distal revascularization interval ligation (DRIL) $(10,11)$ procedure but without the "ligation" step. This circumstance likely prevents the hand from developing "steal" syndrome.

Assessing the palmar arches. Generally DUE is not considered an accurate test to determine intact communicating (deep and superficial) arches (Fig. 2). Likewise, the subjective Allen test is not very reliable to predict hand ischemia after access placement. Palmar arches flow can be seen using DUE but may not define anatomic variations (Fig. 2).

Prior to the Allen test, a complete upper extremities physiological arterial evaluation is performed. The systolic pressure is measured from the upper arm and forearm at the ulnar and radial sites and pulse volume recording (PVR) waveforms are obtained at both levels, bilaterally. Comparison between the two arm pressures and waveforms is necessary. Any pressure gradient $>15 \mathrm{mmHg}$ is considered hemodynamically significant and warrants further investigation (ie arteriogram) to assess the brachiocephalic and subclavian arteries.

The Allen test (4) is performed to evaluate the radial or ulnar artery dominancy while assessing the palmar arches. Photoplethysmography (PPG) probe is used to obtain the thumb arterial flow Doppler signal without any compression maneuvers as a baseline for the test. The signals are obtained repeatedly while manually compressing the radial artery first, the ul- 
nar artery and then both arteries together. With communicating palmar arches arch the signal will maintain a normal amplitude with minimal or no reduction in the amplitude with manual radial or ulnar arterial compression, and may completely disappear with simultaneous radial and ulnar artery compression. The Doppler signal will show remarkable reduction in the amplitude when the dominant artery is compressed, ie both palmar arches do not communicate (Fig. 2). These finding suggests increased risk of ischemia after access placement.

Digital pressures may also be used to evaluate the palmar arches; thumb pressure drop to $50 \mathrm{~mm} \mathrm{Hg}$ or less, while manually compressing either artery at the wrist indicates a risk of steal after HD access placement, especially in an elderly diabetic female.

\section{LOWER EXTREMITY DUE EXAMINATION FOR DIALYSIS ACCESS}

When upper extremity access sites are deemed marginal or ruled out either groin site is to be considered. First, confirm the presence, size and patency of the saphenous vein. A saphenous vein transposition requires a minimum of $30 \mathrm{~cm}$ of healthy vein to allow enough length to create a loop configuration. In cases of inadequate saphenous vein length, a large but short segment of proximal saphenous vein is better suited as venous outflow with an upper thigh PTFE loop graft.

Lower extremity deep venous system is examined for the presence or absence of deep venous thrombosis and physiologic arterial testing is performed with the ankle brachial indices (ABI) obtained to evaluate adequate arterial inflow to the access. The $\mathrm{ABI}$ is calculated by dividing the systolic $\mathrm{BP}$ in the ankle by the higher of the two systolic BPs in the arms. In a normal subject the pressure at the ankle pulses is slightly higher than at the elbow (there is reflection of the pulse pressure from the vascular bed of the feet, whereas at the elbow the artery continues on some distance to the wrist). The ABI is the ratio of the ankle to arm pressure and an ABI of $>0.9$ is considered normal, suggesting that there is no significant peripheral vascular disease affecting the vessels of the legs. A reduced ABI (ABI is normally $>0.9$ bilaterally) is consistent with peripheral artery occlusive disease, with values $<0.8$ indicating moderate diseased and $<0.5$ severe disease. However, a value $>1.3$ is abnormal and suggesting calcification of the walls of the arteries and non-compressible vessels, reflecting severe peripheral vascular disease. Before placing a lower extremity access the iliac arteries and aorta may be assess with DUE for advanced atherosclerotic disease.
Assess the femoral arteries and decide the optimal level of anastomosis site based on the size and presence/absence of atherosclerotic disease and its degree. Right or left side for a thigh access is chosen based on potential future kidney transplant sites. A first time kidney transplant is ideally placed in the right iliac fossa. Therefore, the access is preferred on the left side if vascular anatomy allows. (For the same reason, a PD catheter is optimally placed on the left side in a patient with a potential future first transplant placed in the right iliac fossa).

\section{DUE EXAMINATION OF ESTABLISHED DIALYSIS ACCESS}

Certain signs and symptoms found during the H\&P mandate a DUE examination both in patients being evaluated for the first time access as well as in the case of an established access with problems.

An established access (AVF or PTFE graft) may be assessed for a number of reasons each with a different set of investigative steps (Tab. IV).

It is advisable to have a baseline set of information on all new vascular access. The access is ideally assessed before its use for dialysis. DUE can also be performed at any time after surgery for specific or suspected problems. The extent and content of dialysis access surveillance depends on resources and the technical skills available. The surgeon is often asked if an access is ready to be used for dialysis, although an experienced dialysis nurse is perhaps a better resource about specific access suitability for needle cannulations. Just by physical examination the access quality can be determined with great certainty (ie presence of palpable thrill, the pulse quality and any changes along the access, suggesting intraluminal pathology, ie obstructions). DUE can accurately assess the access diameter, presence of abnormalities (ie size, degree, location, and length of intraluminal stenosis, accessory veins), arterial and venous anastomosis size (diameter) and most importantly volume flow in $\mathrm{mL} / \mathrm{min}$. The baseline access DUE findings will serve as a guide for future interventions.

TABLE IV - INDICATIONS FOR ASSESSING AN ESTABLISHED HEMODIALYSIS ACCESS

1. Post-operative assessment of baseline anatomy and blood flow

2. Cannulation difficulties

3. Abnormal anatomy, ie enlarging aneurysms/pseudoaneurysms

4. Suspected source of infection/abscess

5. Thrombosed access

6. Extremity swelling/edema

7. Hand ischemia-steal 
Access volume flow measurement. With acceptable access anatomy the blood flow rate (in $\mathrm{ml} / \mathrm{min}$ ) becomes the most predictive measure of dialysis access suitability for dialysis cannulation and longevity. Most DUE machines have the capability to calculate access blood flow based on access diameter and mean velocity to derive the volume flow, expressed in $\mathrm{mL} / \mathrm{min}$.

The dialysis machine blood flow range during HD may vary from (low) $180-200 \mathrm{~mL} / \mathrm{min}$ to (medium range) $300-400 \mathrm{~mL} / \mathrm{min}$ up to (high flux) 500 $\mathrm{mL} / \mathrm{min}$. A mature wrist AVF tends to have 500-800 $\mathrm{mL} / \mathrm{min}$, while forearm PTFE loop grafts and brachial artery to vein AVF at the antecubital fossa typically have flow rates in excess of $1000 \mathrm{~mL} / \mathrm{min}$. A dialysis machine flow rate set at $500 \mathrm{~mL} / \mathrm{min}$ may be inadequate and cause the device to alarm from inflow access collapse. The cause of low flow may result from artery stenosis anywhere from the origin of the subclavian artery to the arterial anastomosis. More commonly an anastomotic stenosis of $\leq 2 \mathrm{~mm}$ is found and it may be treated with balloon angioplasty or surgical revision as indicated. Low flow states may also be the result of an outflow (venous) or graft anastomosis stenosis. Outflow stenosis is associated with increased intra-access pressure ("hammer pulse"), often with loss of the "thrill". An intra-access stenotic lesion, often found between aneurysms, can be detected by physical examination. This is felt as a "hammer" pulse proximal and a soft pulse down stream of the obstructive segment. In AVFs a most common pathology is the juxta-anastomotic stenosis located at the first 4$5 \mathrm{~cm}$ from the arterial anastomosis as the vein curves. Flow rates of $\leq 600-800 \mathrm{~mL} / \mathrm{min}$ are associated with poor access survival especially in AV grafts independent of baseline access flow, a significant drop $(25 \%)$ over a limited time (3-4 weeks) is associated with a high failure rate, suggesting underlying developing stenosis. In such a case, fistulogram and balloon angioplasty as indicated is warranted. However, DUE flow measurements may not be superior to an organized clinical assessment, using a formal tracking tool as a surveillance method to prevent hemodialysis access thrombosis (12).

Cannulation difficulties are associated with numerous factors, ie needle puncture skills and experience, access depth (related to obesity), intra-access abnormality (ie hyperplasia, wall adhered thrombus, venous valves), and serpentine winding access conduit. Defining and communicating access anatomy (based on surgery and DUE) to the dialysis unit often prevents or resolves problems.

Aneurysms are commonplace in AVFs and pseudoaneurysms in grafts. Unless cosmetically unacceptable or physically bothersome, corrective measures are taken only when skin viability is threatened. These decisions are based on physical examination. DUE testing does not aid in assessing the aneurysm, but is used to evaluate the overall access prognosis by examining the vascular anastomosis size, flow rate, and outflow vein before any corrective measures are undertaken.

Thrombosed access. DUE will determine the presence and the availability of an adequate outflow vein as well as unexpected pathology. With thrombosed AVFs there is sometimes a short proximal vein segment with some blood flowing through a widely patent arterial anastomosis. This segment can be used as the "arterial" anastomosis in creation of a bypass graft around a worn out, thrombosed, or even infected access into a proximal out flow vein. DUE will delineate possible options in such cases.

Extremity swelling/edema usually occurs as a complication of central vein obstruction due to previous dialysis catheters or lines. As part of the access surveillance examining the subclavian vein may suggest a stenosis or a more proximal obstruction from an abnormal flow pattern (lack of flow phasisity and pulsatility) or lack of augmentation upon compressing on the arm. Confirmation and treatment of suspected central venous narrowing/occlusion requires a venogram and corrective interventional radiology procedures as indicated.

Hand ischemia or so-called "steal" represents is a severe and somewhat unpredictable complication after and occurs in 6-10\% of AV graft and upper arm AVFs but rarely with wrist AVFs $(<1 \%)$. Detailed history and physical examination is quite accurate in confirming the diagnosis. The vascular laboratory digital pressure determination helps quantify the degree of severity. Partial manual compression of the access helps to determine the degree of correction (restoration) possible with banding (13) or the more extensive DRIL procedure $(10,11)$, or with proximalization of the arterial inflow (PAI) anastomosis (14) (Fig. 2).

Manual compression of the access will relieve the patient's symptoms (ie pain tingling) in cases of acute ischemia, again confirming the diagnosis. Sonogram examination of the proximal artery will direct further diagnostic accuracy and treatment options since inflow obstruction from proximal arterial stenosis is present up to one-third of cases. If suspected by DUE, arteriogram and balloon angioplasty of the proximal inflow artery is the next logical step.

\section{When can a dialysis access be used?}

There is much variation and difference in opinions and policies. HD access suitability for cannulation can effectively be assessed (physical examination) by 
an experienced dialysis nurse or technologist. Before using the access a baseline DUE assessment with volume flow measurement is advised. Table II lists the minimal requirements for a useable dialysis access. Wrist AVF. A wrist (radial) artery diameter of $\leq 2.5$ $\mathrm{mm}$ is generally associated with inadequate fistula flow $(<500 \mathrm{~mL} / \mathrm{min})$, ie lack of maturation and eventually fistula failure. Likewise, an AV anastomosis diameter of $\leq 2.5 \mathrm{~mm}$ will likely yield an inadequate flow rate $(4,5)$ (Tab. II).

In contrast to PTFE grafts AVFs are less predictable and associated with slower and delayed maturation of 4-8 weeks. In our experience $30-40 \%$ of wrist fistulae do not develop to be used and require revisions or placement of new access. This in agreement with the recent multicenter study reporting a $60 \%$ clinical maturation failure rate (9). Early aggressive diagnostic and corrective measures are advised. Due to the often smaller size vessels and difficult anatomy (most commonly obesity), cannulation difficulties and complications such as infiltration and hematoma formation are common. In general at least weeks should be allowed for maturation of any AVF. On the other hand, a non-obese person with excellent vascular anatomy may have a fistula ready to be cannulated within days to 1-2 weeks after surgery; in the situation of an urgent need for dialysis, rather than placing a cuffed CVC, this early use of a fistula is warranted. In such a case the "best sticker" policy is advised. However, in general it is suggested to wait 3-4 weeks allowing the fistula vein to increase in size and become "arterialized". Characteristics of a mature/useable AVF include (15):

- A cannulation segment of 15-20 cm, allowing for needle puncture site rotation.

- A consistent diameter of $\geq 6 \mathrm{~mm}$.

- A blood flow rate of at least 500-600 mL/min.

Upper Arm AVF. This is typically a brachial artery to cephalic vein at or above the antecubital fossa. Same rules apply as with wrist $\mathrm{AVF}$, although flow rates tend to be higher. One peculiar common anatomic abnormality is a stenosis at the cephalic-subclavian vein junction. The awareness of the "cephalic arch" stenosis is important to the DUE examiner. With the availability of ultra-high pressure angioplasty balloon devices, effective dilatation has become more consistent $(16,17)$.

Transposed basilic and cephalic veins. Assuming adequate baseline DUE assessment, it is advised (as with graft material) to wait 3-4 weeks while the vein is incorporated with surrounding tissue $(5,16,18)$. PTFE grafts. Graft maturation is more predictable and can consistently be used for HD after 2-4 weeks, although in the author's opinion 3-4 weeks is preferred to allow solid incorporation by surrounding tissue. Assessment criteria include degree of perigraft tissue edema, redness, pulse quality, and presence of a palpable thrill. A baseline DUE with volume flow is advised.

In all patients with an upper extremity new access it is recommended to make hand fists over a soft ball to minimize arm swelling. Elevation and placing icepacks for 12-24 hr after surgery on PTFE graft sites markedly prevents swelling and decreases postoperative pain (15). The use of arm slings is strongly advised against.

Portable ultrasound devices. Portable ultrasound devices are extremely valuable tools to determine the exact anatomy, and guide the needle for placement of catheters as recommended by the DOQI guidelines (15). The right internal jugular vein is the preferred site for dual lumen cuffed, tunneled catheter insertion. With increased experience, the use of disposable needle guides may be omitted or bypassed, which saves time and simplifies insertion. It is strongly recommended using ultrasound guidance for all large vein access interventional procedures (19). Neck vascular abnormal anatomy is encountered in $30 \%$ of cases $(18,20)$. A portable ultrasound device should be used for dialysis needle punctures for training purposes and in all cases with known and predicted anatomical difficult access cannulations.

\section{ACKNOWLEDGEMENTS}

The authors acknowledge the help of Stephen T. Brown, MA, Medical Illustrator, Dallas TX, for drawing the figures with professional skills.

Conflict of interest statement: none declared.

Address for correspondence:

Ingemar Davidson, $M D, P h D, F A C S$

Division of Transplant

Department of Surgery

Parkland Memorial Hospital University of Texas Southwestern

Medical Center

5939 Harry Hines Blvd

Dallas, TX 75390-8567

USA

drd@ingemardavidson.com 


\section{REFERENCES}

1. Davidson I, Gallieni M, Saxena R, Dolmatch B. A patient centered decision making dialysis access algorithm. J Vasc Access 2007; 8: 59-68.

2. Beathard GA. Physical examination: the forgotten tool. In: Gray R, Sands J eds. A Multidisciplinary Approach for Hemodialysis Access. New York: Lippincott Williams \& Wilkins, 2002; 111-8.

3. Davidson IJA. Access for Dialysis: Surgical and Radiologic Procedures. 2nd edn. Landes Bioscience, 2002.

4. Kamienski RW, Barnes RW. Critique of the Allen test for continuity of the palmar arch assessed by Doppler ultrasound. Surg Gynecol Obstet 1976; 12: 861-4.

5. Davidson IJ, Cava-Bartsch C. Basilic vein transposition. A case report with contra-lateral removal of a large A-V fistula. J Vasc Access 2005; 6: 49-61.

6. Tordoir JHM, de Bruin HG, Hoeneveld H, Eikelboom BC, Kitslaar PJ. Duplex ultrasound scanning in the assessment of AV fistulae created for hemodialysis access: Comparison with digital subtraction angiography. J Vasc Surgery 1989; 10 122-8.

7. Chao AKH, Daley T, Gruenwald S, et al. Duplex ultrasound criteria for the assessment of stenoses in radiocephalic hemodialysis fistulae. J Vasc Technology 2001; 25: 203-8.

8. Weiswasser JM, Kellicut D, Arora S, Sidawy AN. Strategies of arteriovenous dialysis access. Semin Vasc Surg 2004; 17: 10-8.

9. Beck G, Dember L, Feldman H. The Dialysis Access Consortium (DAC) Study Group. The Dialysis Access Consortium (DAC) trial of clopidogrel for prevention of early thrombosis of hemodialysis AV fistulasprimary results: Presented at ASN Annual Meeting, San Francisco, 4 November 2007. San Francisco, CA.

10. Berman SC, Gentile AT, Glickman MH, et al. Distal revascularization-interval ligation for limb salvage and maintenance of dialysis access in ischemic steal syndrome. J Vasc Surg 1997; 26:393-404.

11. Knox RC, Berman SC, Hughes JD, Gentile AT, Mills JL. Distal revascularization-interval ligation: A durable and effective treatment for ischemic steal syndrome after hemodialysis access. J Vasc Surg 2002; 36: 250-6.

12. Schuman E, Ronfeld A, Barclay C, Heinl P. Comparison of clinical assessment with ultrasound flow for hemodialysis access surveillance. Arch Surg 2007; 142: 1129-33.

13. Davidson IJA. Access for Dialysis: Surgical and Radiologic Procedures. 2nd edn. Landes Bioscience, 2002; 20.

14. Zanow J, Kruger U, Scholtz H. Proximalization of the arterial flow: A new technique to treat access related ischemia. J Vasc Surg 2006; 43: 1216-21.

15. National Kidney Foundation. K/DOQI clinical practice guidelines for vascular access update 2006. Am J Kidney Dis 2006; 48(Suppl 1): S176-322.

16. Turmel-Rodrigues L, Pengloan J, Baudin S, et al. Treatment of stenosis and thrombosis in hemodialysis fistulas and grafts by interventional radiology. Nephrol Dial Transplant 2000; 15: 2029-36.

17. Rajan DK, Clark TWI, Patel NK, Stavropoulos SW, Simons ME. Prevalence and treatment of cephalic arch stenosis in dysfunctional autogenous hemodialysis fistulas. J Vasc Interv Radiol 2003;14: 567-73.

18. Silva MB Jr, Hobson RW 2nd, Pappas PJ, et al. Vein transposition in the forearm for autogenous hemodialysis access. J Vasc Surg 1997; 26: 981-8.

19. Gallieni M. Central vein catheterization of dialysis patients with real time ultrasound guidance. J Vasc Access 2000; 1: 10-4.

20. Denys BG, Uretsky BF. Anatomical variations in internal jugular vein location: Impact on central venous access. Crit Care Med 1991; 19: 1516-9. 